\title{
Minimal formulation of the linear spatial analysis of capillary jets: Validity of the two-mode approach
}

\author{
H. González* and P. A. Vazquez \\ Departamento de Física Aplicada III, Escuela Técnica Superior de Ingeniería, Universidad de Sevilla, \\ Camino de los Descubrimientos, s/n, 41092 Sevilla, Spain \\ F. J. García \\ Departamento de Física Aplicada I, Escuela Politécnica Superior, Universidad de Sevilla, \\ c/ Virgen de África, 7, 41011 Sevilla, Spain \\ J. Guerrero \\ Department of Chemistry and Physics, Augusta University, 1120 15th Street, SCI W3005, \\ Augusta, Georgia 30912, USA
}

(Received 21 February 2017; published 18 April 2018)

\begin{abstract}
A rigorous and complete formulation of the linear evolution of harmonically stimulated capillary jets should include infinitely many spatial modes to account for arbitrary exit conditions [J. Guerrero et al., J. Fluid Mech. 702, 354 (2012)]. However, it is not rare to find works in which only the downstream capillary dominant mode, the sole unstable one, is retained, with amplitude determined by the jet deformation at the exit. This procedure constitutes an oversimplification, unable to handle a flow rate perturbation without jet deformation at the exit (the most usual conditions). In spite of its decaying behavior, the other capillary mode (subdominant) must be included in what can be called a "minimal linear formulation." Deformation and mean axial velocity amplitudes at the jet exit are the two relevant parameters to simultaneously find the amplitudes of both capillary modes. Only once these amplitudes are found, the calculation of the breakup length may be eventually simplified by disregarding the subdominant mode. Simple recipes are provided for predicting the breakup length, which are checked against our own numerical simulations. The agreement is better than in previous attempts in the literature. Besides, the limits of validity of the linear formulation are explored in terms of the exit velocity amplitude, the wave number, the Weber number, and the Ohnesorge number. Including the subdominant mode extends the range of amplitudes for which the linear model gives accurate predictions, the criterion for keeping this mode being that the breakup time must be shorter than a given formula. It has been generally assumed that the shortest intact length happens for the stimulation frequency with the highest growth rate. However, we show that this correlation is not strict because the amplitude of the dominant mode has a role in the breakup process and it depends on the stimulation frequency.
\end{abstract}

DOI: 10.1103/PhysRevFluids.3.044802

\section{INTRODUCTION}

Capillary jets have historically attracted much attention of researchers because of both their practical use in technology and industry $[1,2]$ and their richness in behavior as a physical system (see

\footnotetext{
*helio@us.es
} 
Refs. [3,4] for a review of the main bibliography and advances in this field). They also constitute a remarkable example of success of predictions of linear models, in spite of necessarily having a final strongly nonlinear evolution associated with the breakup. However, some basic aspects are poorly handled by practitioners and researchers. Specifically, the connection between the conditions at the exit and the breakup length are still subject to mistakes. In subsequent works to those of Rayleigh $[5,6]$, it was usual to disregard the contribution of exponentially decaying solutions to the linear problem when compared with that of the exponentially growing mode. Although this assumption is valid in many experimental situations, its consequences are negative in two ways: (i) It prevents explaining the nonexponential initial transients found in experiments and numerical simulations and (ii) it makes impossible to handle pure impulsive initial conditions, prescribing the initial velocity perturbation of the jet [7].

We can find in the literature several attempts to describe the breakup length as a function of impulsive conditions, some of them very recent $[8,9]$, where the procedure is to look for an equivalent pure deformation condition. In García and González [7], the authors clarified this question. They formulated a temporal linear estimate of the breakup time (proportional to the breakup length) for any kind of initial conditions. The assumptions of that work were the following: (i) a temporal modal analysis is able to describe the linear evolution of the jet, (ii) the two relevant conditions at the exit are the shape deformation and the mean axial velocity over the jet section, and (iii) the continuity equation integrated on a slice allows to build a reduced model with only the two capillary modes, the dominant one (growing for wave numbers below the Rayleigh cutoff limit) and subdominant one (decaying for all wave numbers). These modes are the only ones having non-negligible flow rate. The remaining, infinitely many hydrodynamic modes are recirculating and have negligible shape deformation. In this way, the resulting model captured the essential features of the capillary instability and reduced the number of modes of the boundary-value problem from infinity to only two. The conclusions were that the contribution of the subdominant capillary mode, on the one hand, explains the nonexponential initial transients and, on the other hand, allows us to formulate general initial conditions with both initial deformation and velocity perturbation.

The temporal approach to the linear evolution of capillary jets is accurate for not very low Weber numbers, as Keller and coworkers demonstrated by adopting a spatial approach [10]. Their linear theory is valid for any jet velocity greater than a critical value under which the jet exhibits absolute instability [11]. The spatial analysis reveals a set of modes richer than the one from the temporal analysis, and it is primarily classified in modes living downstream and upstream from any jet station [12]. Among the downstream modes generated at the jet exit, some modes (the two capillary modes and the hydrodynamic modes) are the spatial counterparts of the temporal modes, and others (the inertial modes) are genuinely spatial. Although this detailed modal analysis formally allows relating the exit conditions with the subsequent evolution of the jet, this task has not been carried out.

The main aim of the present work is to find a reduced model to account for arbitrary exit conditions through a procedure similar to that in García and González [7], but starting from the mentioned set of downstream spatial modes. In this way, our results will be valid for a wider range of Weber numbers. These findings correct previous attempts found in the literature [8,9].

It would be desirable to compare these linear results with experiments. However, real jets have exit velocity profiles dependent on the nozzle geometry and wettability properties: the profiles are not uniform in general. Only after a process of viscous relaxation taking place over distances of the order of the Reynolds number times the jet radius [13], the velocity profile becomes almost uniform and the jet radius reaches an almost constant value. These complications can obscure the actual performance of the linear models here presented when compared with experiments. This is the reason why we have included numerical simulations with idealized exit conditions, namely, plug velocity profiles for the basic flow. Notwithstanding the foregoing, jets emanating from thin orifices have almost plug velocity profiles. Experiments conducted under these conditions with water jets of some $1 \mathrm{~mm}$ in diameter, harmonically stimulated close to the maximum of the Rayleigh dispersion relation, resulted in measured breakup lengths that agree with the linear predictions [14]. 
In this work, we present the spatial, two-mode theory in Sec. II, the description of numerical essays using Gerris in Sec. III, results and comparison between theory and nonlinear numerical simulations in Sec. IV, and discussion and criticisms about previous literature in Sec. V.

\section{TWO-MODE SPATIAL DESCRIPTION OF THE JET EVOLUTION}

Consider the discharge of a liquid in a gas through a circular nozzle that forms an axisymmetric jet with an idealized plug velocity profile of magnitude $v$. Assume that the orifice has a sharp edge to which the liquid is pinned, in order to avoid unwanted wetting phenomena. Also neglect the action of gravity and the interaction with the outer gas, so that the liquid is only subject to capillary and viscous forces. The required parameters for the physical description are the liquid density $\rho$, its dynamic viscosity $\mu$, and its surface tension $\gamma$. The fluid-dynamic problem admits a basic solution consisting in a cylindrical jet with the same radius and exit velocity profile at any flow station. Convenient scales in systems governed by capillarity are the jet radius, $R$, for lengths, the capillary time, $t_{c} \equiv\left(\rho R^{3} / \gamma\right)^{1 / 2}$, for time, and the capillary velocity, $v_{c} \equiv R / t_{c}$, for velocities. In what follows, we will deal with dimensionless quantities.

Let us now impose a perturbation at the exit, harmonic in time. After a transient, the system evolves to a permanent regime also harmonic in time, with the same imposed frequency, disregarding very small fluctuations visible in the breakup region (see Sec. III). According to Guerrero et al. [12], the perturbations in radius and axial velocity at any axial position $z$ can be constructed as a combination of spatial modes, but not all of them will be necessary for our analysis. There are modes propagating upstream and downstream from this position. As the stimulation takes place at the jet exit, it would be expected that only those modes defined downstream from the exit are required. Recently, Umemura [15] proposed that some modes propagating upstream from the breakup region can produce a self-stimulation of the jet, independent of the forced stimulation that we are considering. However, its predictions and observations restrict this phenomenon to jets with low Weber numbers and without forcing. We assume that this is not our case. In principle, for an arbitrary perturbation in the velocity at the jet exit, the complete infinite set of downstream modes would be necessary. This set includes two capillary modes, and the infinite families of hydrodynamic and inertial modes. Every mode in these infinite families have negligible net flow rate across the cross section of the jet, due to its recirculating nature in the case of the hydrodynamic family and to its strong spatial decay for the inertial family. For this reason, it is not surprising that all modes in these families have also a negligible surface deformation associated to them. It is physically reasonable to expect that any mode without associated deformation has a very weak influence over the jet breakup. Consequently, it is advantageous to formulate a reduced description of the flow in terms of the surface radius, $F(z, t)$, and the mean axial velocity perturbation over the jet cross section, $W(z, t)$, and combine the two capillary modes to describe the perturbation, i.e., the only modes for which these magnitudes are non-negligible. Specifically, we can write

$$
F(z, t)=1+\operatorname{Re}\left[f(z) e^{-i \omega t}\right] ; W(z, t)=\beta+\operatorname{Re}\left[w(z) e^{-i \omega t}\right],
$$

where $\omega$ is the dimensionless perturbation frequency and $\beta \equiv v t_{c} / R=v(\rho R / \gamma)^{1 / 2}$ is the square root of the Weber number. The quantities $f(z)$ and $w(z)$ are complex in general and, as mentioned, they will be constructed as

$$
\begin{gathered}
f(z)=f_{\mathrm{d}} e^{i k_{\mathrm{d}} z}+f_{\mathrm{s}} e^{i k_{\mathrm{s}} z}, \\
w(z)=w_{\mathrm{d}} e^{i k_{\mathrm{d}} z}+w_{\mathrm{s}} e^{i k_{\mathrm{s}} z},
\end{gathered}
$$

where $k_{\mathrm{d}}$ and $k_{\mathrm{s}}$ are the complex wave numbers associated to the capillary dominant and capillary subdominant modes, respectively. Both are solutions to the dispersion relation obtained from the spatial stability analysis of the jet $[11,12]$ (in the latter reference, the time dependence is actually the same as in the text, but it appears with the typographic error $\left.e^{i \omega t}\right) . k_{\mathrm{d}}$ and $k_{\mathrm{s}}$ depend on the stimulation 
frequency, the Weber number, and the Ohnesorge number, $\mathrm{Oh} \equiv \mu /(\rho R \gamma)^{1 / 2}$. Their real parts, $k_{\mathrm{d}, r}$ and $k_{\mathrm{s}, r}$, give the spatial periodicity; the opposite of their imaginary parts, $-k_{\mathrm{d}, i}$, and $-k_{\mathrm{s}, i}$, give their spatial growth rate. The complex amplitudes $f_{\mathrm{d}}, f_{\mathrm{s}}, w_{\mathrm{d}}$, and $w_{\mathrm{s}}$ must be determined from the conditions at the exit, $z=0$, as explained below.

The key to construct our reduced model is to relate deformation and mean axial velocity through a mass balance over a slice of the jet:

$$
\frac{\partial F^{2}}{\partial t}+\frac{\partial F^{2} W}{\partial z}=0
$$

It is important to realize that this is not a new equation but a convenient synthesis of the mass conservation and the kinematic condition, which are parts of the full set of governing equations (see Guerrero et al. [12]). The reader familiar with one-dimensional models of capillary jets can recognize this equation as the one accounting for the mass conservation without any approximation [16].

Assuming small departures from the basic flow, i.e., $|f(z)| \ll 1$ and $|w(z)| \ll 1$, introducing (1) into (4) and linearizing, we arrive to

$$
-i \omega f+\beta \frac{d f}{d z}+\frac{1}{2} \frac{d w}{d z}=0 .
$$

Substituting (2) and (3) in it and requiring validity for arbitrary $z$, we obtain the relations

$$
w_{\mathrm{d}}=2 \frac{\omega-\beta k_{\mathrm{d}}}{k_{\mathrm{d}}} f_{\mathrm{d}}, \quad w_{\mathrm{s}}=2 \frac{\omega-\beta k_{\mathrm{s}}}{k_{\mathrm{s}}} f_{\mathrm{s}} .
$$

In this way, we have reduced the unknowns to two complex numbers, $f_{\mathrm{d}}$ and $f_{\mathrm{s}}$. If we provide at the exit the radius perturbation, $f_{0}$, and the mean axial velocity perturbation, $w_{0}$, we can determine these unknowns. Note that these exit conditions are in general complex, as they have amplitude and phase. By putting $f(0)=f_{0}$ and $w(0)=w_{0}$, and solving for $f_{\mathrm{d}}$ and $f_{\mathrm{s}}$, we find

$$
\begin{aligned}
& f_{\mathrm{d}}=\frac{-k_{\mathrm{d}} k_{\mathrm{s}}}{2 \omega\left(k_{\mathrm{d}}-k_{\mathrm{s}}\right)}\left[w_{0}+2\left(\beta-\frac{\omega}{k_{\mathrm{s}}}\right) f_{0}\right], \\
& f_{\mathrm{s}}=\frac{k_{\mathrm{d}} k_{\mathrm{s}}}{2 \omega\left(k_{\mathrm{d}}-k_{\mathrm{s}}\right)}\left[w_{0}+2\left(\beta-\frac{\omega}{k_{\mathrm{d}}}\right) f_{0}\right],
\end{aligned}
$$

which give the amplitudes of the capillary modes for any possible initial conditions at the exit in terms of surface deformation and mean axial velocity. The simple algebra required to arrive to this result, based on Eq. (4), could be contrasted with the cumbersome procedure needed if we had (i) retained all the spatial modes, (ii) applied the exit conditions to the original formulation, and finally (iii) calculated an integral over the jet section to keep only the modes with significant deformation and net flow rate.

By putting either $f_{0}=0$ or $w_{0}=0$ in these formulas, we can study the linear evolution and estimate the breakup length for pure-impulse or pure-deformation exit conditions, respectively, which are particularly interesting from an experimental viewpoint. We can also discuss the question of the equivalence between impulse and deformation perturbations by merely comparing both contributions to the amplitude of the capillary dominant mode, $f_{\mathrm{d}}$. The equivalence takes place between perturbations satisfying $\left|w_{0}\right|=2\left|\left(\beta-\omega / k_{\mathrm{s}}\right) f_{0}\right|$.

The formulas (7) and (8) extend the findings obtained from the temporal analysis in García and González [7] to arbitrary values of the Weber number. In fact, we must expect that they agree in the limit $\beta \rightarrow \infty$. To prove it, recall that a first approximation for $\beta \gg 1$ is

$$
k_{\mathrm{d}} \simeq k_{\mathrm{temp}}-i \frac{\alpha_{\mathrm{d}}}{\beta}, \quad k_{\mathrm{s}} \simeq k_{\mathrm{temp}}-i \frac{\alpha_{\mathrm{s}}}{\beta},
$$

where $k_{\text {temp }} \equiv \omega / \beta$ is the real dimensionless wave number used in the temporal stability analysis, $\alpha_{\mathrm{d}}$ is the corresponding dominant temporal growth rate (positive for $0<k_{\text {temp }}<1$ ), and $\alpha_{\mathrm{s}}$ is the 


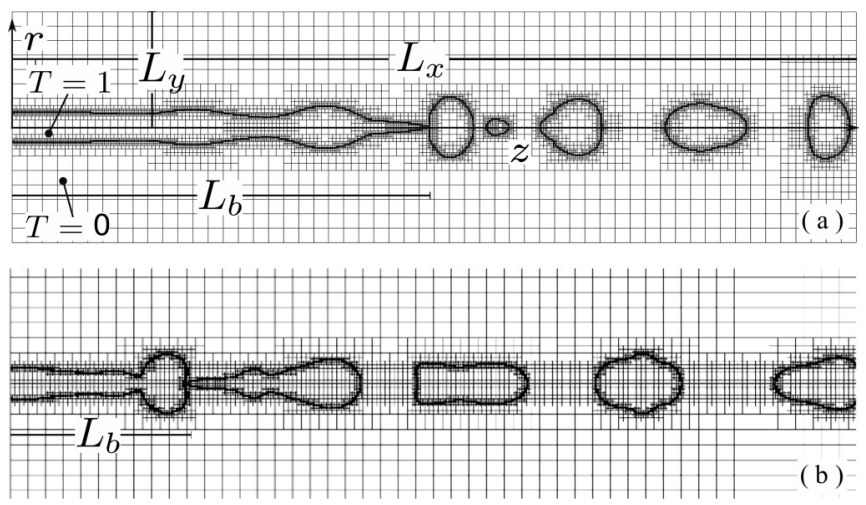

FIG. 1. (a) Computational geometry, boundary conditions for the tracer, and typical profile of a jet just before the front-ligament pinching (FLP), with the refined quadtree mesh. The lower half of the figure is a reflection of the upper half, as the computations are axisymmetric. For this simulation it is $\mathrm{We}=14.8, \mathrm{Oh}=$ $0.01, k_{\text {temp }}=0.697$, and $w_{0}=0.154$. (b) An example of rear-ligament pinching (RLP).

subdominant one (negative for the same range of wave numbers). Introducing these approximations in (7), we have, to leading order in $\beta^{-1}$ and keeping $k_{\text {temp }}$ fixed

$$
f_{\mathrm{d}} \simeq \frac{-1}{\alpha_{\mathrm{d}}-\alpha_{\mathrm{s}}}\left[\frac{i k_{\mathrm{temp}}}{2} w_{0}+\alpha_{\mathrm{s}} f_{0}\right]
$$

and similarly for $f_{\mathrm{s}}$. This is the expected limit that can be found in García and González [7].

In order to have an estimate of the breakup length, we can apply the linear theory through the condition $\operatorname{Max}_{t} f\left(L_{b}, t\right)=1$, where we consider, at each position $z$, the maximum value of the deformation for any time $t$, and select $L_{b}$ as the first value of $z$ able to achieve this condition. A customary practice is to neglect the subdominant component of the shape evolution owing to its decay. Then, the breakup length, here called $L_{b}^{(1)}$, can be explicitly estimated as

$$
L_{b}^{(1)}=-\frac{1}{k_{\mathrm{d}, i}} \ln \left|\frac{w_{0} k_{\mathrm{d}} k_{\mathrm{s}}}{2 \omega\left(k_{\mathrm{d}}-k_{\mathrm{s}}\right)}\right| .
$$

However, if the jet is short due to a strong stimulation, the subdominant mode can have a significant role, as we will show in Sec. IV.

\section{NUMERICAL SIMULATIONS}

In order to check the performance of our linear model, we present numerical simulations starting with the same basic flow, i.e., with cylindrical shape and plug axial velocity profile, as done by Moallemi et al. [8]. Numerical simulations have the advantage of avoiding complications associated with relaxation processes of the axial velocity profile at the exit of a nozzle, very dependent on its geometry and usually leading to a change in radius $[13,17]$. We have performed the numerical simulations with GERRIS [18]. This is a open-source code developed by Popinet. It implements a quad/octree spatial discretization that allows refining the mesh locally. The interface is tracked with a volume of fluid (VOF) scheme. The Navier-Stokes equations are solved with a fractional-step projection method. As it is shown in the given reference, this code is able to reproduce with a great accuracy the breakup of a capillary jet. For more technical details, the reader can refer to Popinet [18].

The flow is assumed to be axisymmetric, a reasonable restriction in the laminar regime that substantially reduces the computation effort. Figure 1 shows the geometry and results for a typical simulation. This figure is made from the original output of the simulation juxtaposed to a mirrored 
image. The geometry is a rectangle of dimensions $L_{x} \times L_{y}$. The liquid enters the domain through the left border. A scalar tracer is defined, $T$, so that its value is $T=1$ in the liquid phase and $T=0$ in the gaseous phase. The physical properties of the gas phase are those of air, while for the liquid phase we selected the density and surface tension of water at ambient temperature, but various values for the viscosity. The conditions for the tracer on the left boundary are shown in the figure. On the remaining boundary, a Neumann condition is imposed. For the velocity, we impose an entrance normal profile with a time-dependent perturbation where $T=1$, while we set $u=0$ where $T=0$. On the rest of the boundary, an open condition is applied. In all the simulations, the radius of the entrance is 1 , $L_{y}=8$, and $L_{x}$ is between 48 and 160, large enough to not influence the breakup of the jet.

As is evident in Fig. 1, and in experiments [3], in general there are a main drop and a ligament, the latter leading to a satellite drop. Accordingly, there are two possible pinching events: front-ligament pinching, FLP (downstream the ligament, as in the figure), and rear ligament pinching, RLP (upstream the ligament), which lead to two breakup lengths, slightly different in general. In some cases, only one type of pinching occurs and there are no satellites. Concerning the temporal evolution of that ligament, the order in which both types of pinching takes place may change. Our numerical simulations have revealed a variety of pinching behaviors when changing the relevant parameters. In contrast, the linear theory gives only one breakup length because it does not predict the formation of a satellite drop.

The mesh is refined locally using the gradient of the tracer function as the refinement criteria. We have refined down to level 7 in GERRIS. This value implies a minimum cell size of 0.0625 times the radius of the jet. Then, the spatial resolution is about half that value, taking into account that the VOF scheme reconstructs the interface inside each cell. Further refinements do not change significantly the results for the breakup length. In the literature, we can find works where the developed codes were able to accurately describe values of the radius of the filament as small as $10^{-6}$ times the radius of the jet [19] and, in this way, they validated the scaling and pinch-off predictions [20]. But in this study our goal is to assess the capability of the two-mode model to predict the breakup length, not to describe in detail the breakup process. The breakup length $L_{b}$ is obtained from postprocessing the outcome of the simulation. At each time step, the interface is saved and, later, the position of the transitions from liquid to air along the symmetry axis are obtained, producing plots similar to those shown in Fig. 2. Figure 2(a) corresponds to a case with only one kind of pinching (FLP), while 2(b) one shows the two kinds of pinching (FLP and RLP). In a numerical simulation, we observe several consecutive pinchings of the jet, almost, but not strictly periodic, and we take the average of the breakup lengths for each kind of pinching. The number of consecutive observed pinchings for each numerical simulation ranges from 5 to 10 [see, for instance, the encircled pinchings in Fig. 2(b)]. The dispersion bar of a set of pinching events depends on the parameters of the simulation, especially on the amplitude the perturbation, but in most cases it is inappreciable when represented in our plots. Only when the frequency of the perturbation is not close to the value of maximum growth or the Ohnesorge number is of the order of unity, the dispersion bar can become visible.

\section{RESULTS}

We have restricted ourselves to pure impulsive exit conditions because they are the most usual in experiments and applications. However, our analysis is straightforwardly extensible to the pure deformation exit conditions, or to the general case in which perturbations both in jet radius and mean axial velocity coexist.

Our reduced model disregards the effect of all the spatial modes but the two capillary modes, arguing that the former modes have neither net flow rate nor shape deformation associated with them. To validate this approach, a numerical simulation has been designed, consisting of determining the breakup length of a jet exiting with different $r$-dependent axial velocity perturbations, but with the same perturbed flow rate. The selected profiles for the test are the plug profile, $w(r)=w_{0}$, a parabolic profile, $w(r)=2 w_{0}\left(1-r^{2}\right)$, and finally a U-shaped profile, $w(r)=21 w_{0}\left(r^{4}-r^{5}\right)$. We insist that all three velocity profiles correspond to the perturbation, not to the basic flow (which is 

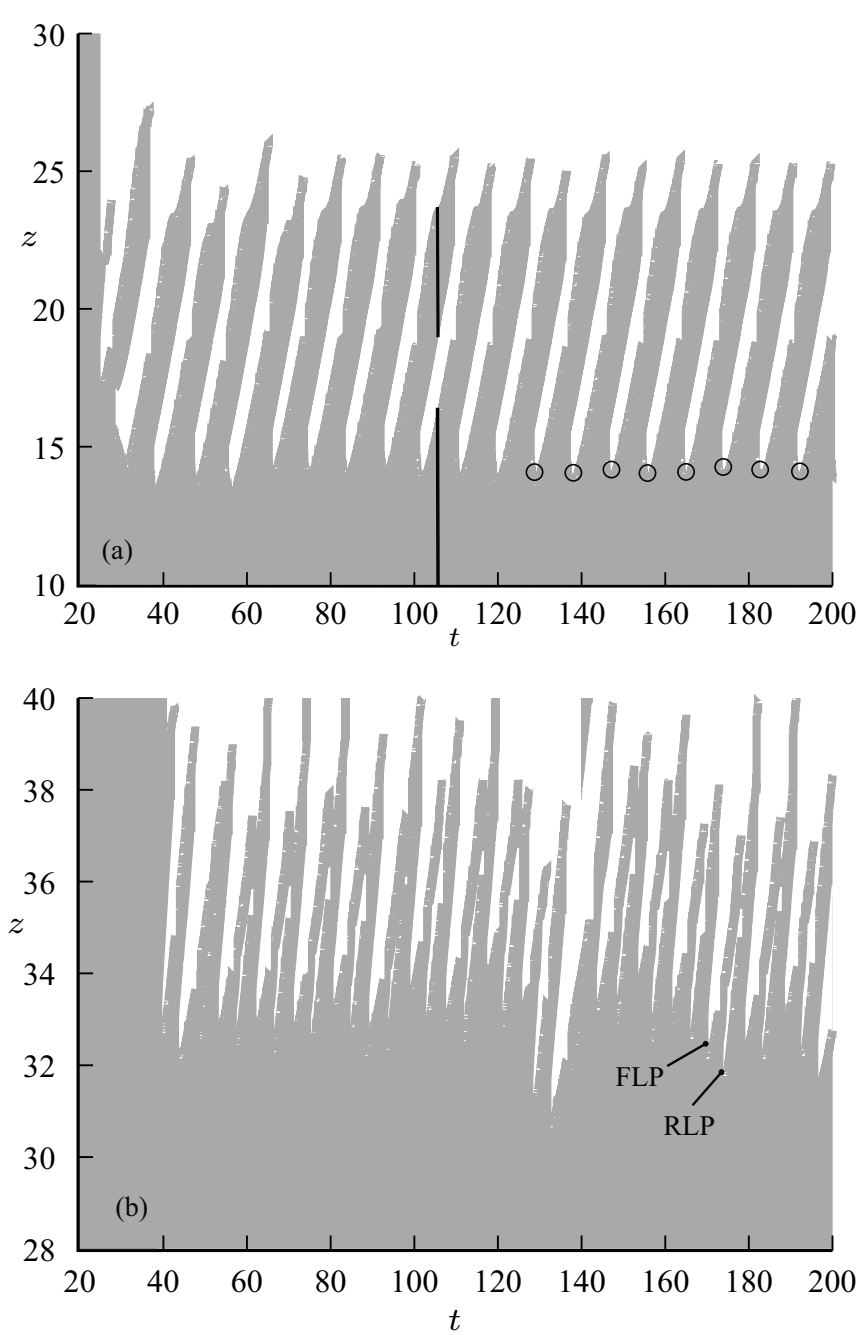

FIG. 2. Breakup diagrams showing for each dimensionless time, $t$, the parts of the axis occupied by the liquid (filled) and by the air (empty). The simulation has been carried out for $\mathrm{Oh}=0.01, k_{\text {temp }}=0.697, w_{0}=0.154$, and (a) $\mathrm{We}=4$ or (b) $\mathrm{We}=20$. To help its interpretation, in panel (a) two vertical segments are drawn for a specific instant, the lower one in $z$ belonging to the intact length of the jet and the upper one defining the limits of the most recently detached drop. All the previously detached drops have been erased for the sake of clarity. In the diagram (a), after the transient regime, eight breakup events (encircled) have been selected to calculate the mean breakup length and its associated dispersion. In diagram (b), examples of FLP and RLP are marked.

always a plug velocity profile), and that they have the same associated perturbation in flow rate. The numerical results, summarized in Fig. 3, show that the influence of the perturbation velocity profile on the breakup length is below $3 \%$ for these representative values of parameters.

A basic check of the performance of the linear theory is to compare its predictions for the breakup length with the numerical simulations findings. Figure 4 shows the breakup length versus the amplitude of the harmonic velocity perturbation in the pure impulsive case, $w_{0}$. The remaining parameters are fixed to the values $\mathrm{We}=14.8, \mathrm{Oh}=0.01$, and $k_{\text {temp }}=0.697$, the latter corresponding to the maximum of the Rayleigh curve. We also represent the front-ligament and rear-ligament pinching positions for each numerical essay; in all cases, the first pinching in time is the closest 


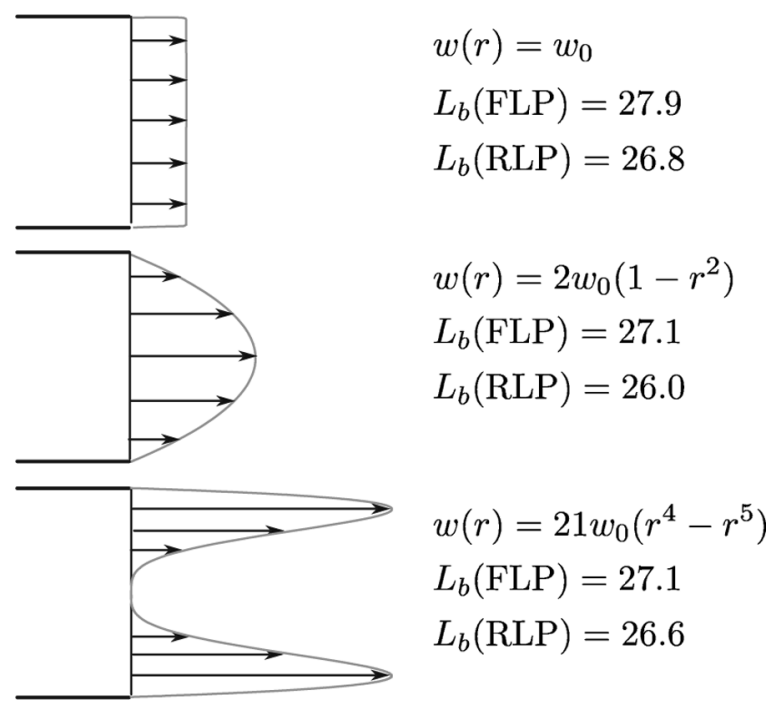

FIG. 3. Numerical estimates of breakup lengths for different velocity perturbation profiles, all having the same flow rate. Breakups for front-ligament pinching (FLP) and rear-ligament pinching (RLP) are presented. The simulations are conducted for $w_{0}=0.154$, We $=14.8, \mathrm{Oh}=0.01$, and $k_{\text {temp }}=0.697$. Deviations in the measured lengths over several breakup processes are estimated as \pm 0.2 .

to the theoretical curve. Notice that in this semilogarithmic representation, the one-mode spatial breakup length is a straight line with slope $-1 / k_{\mathrm{d}, i}$, according to Eq. (11). The inclusion of the subdominant mode into the theoretical prediction makes it deviate from a straight line and brings it closer to the numerical values.

To have an idea of when the two modes are necessary to have a good estimate for the breakup length, a simple formula can be found in the following way: define a small parameter $p$ measuring the relative contribution of the subdominant mode to the jet perturbation $f(z, t)$ by $\exp \left(k_{\mathrm{d}, i}\right)=p \exp \left(k_{\mathrm{s}, i}\right)$ and insert in the breakup condition $f\left(L_{b}, t\right)=1$. Next, write the breakup length as $L_{b}=L_{b}^{(1)}+\Delta L_{b}$,

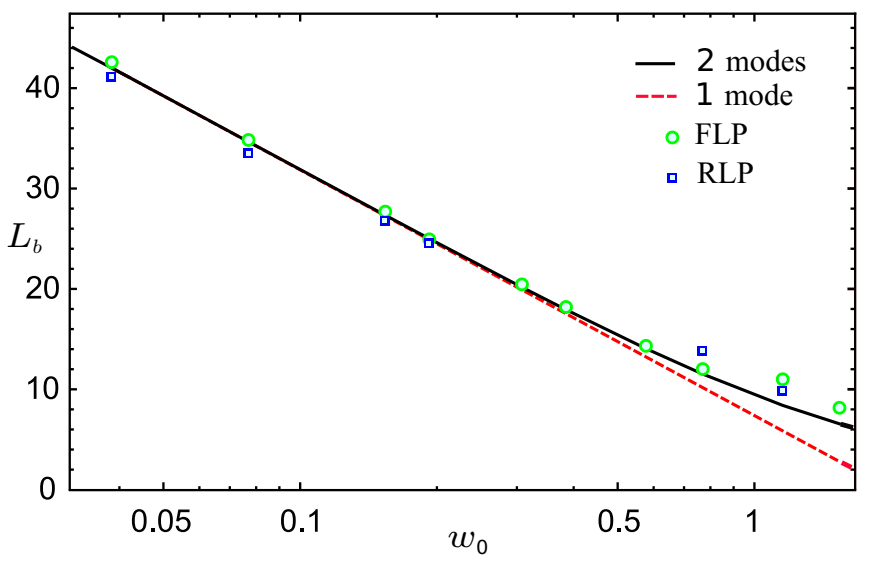

FIG. 4. Dimensionless breakup length versus the amplitude of perturbation of the mean axial velocity, for $\mathrm{We}=14.8, \mathrm{Oh}=0.01$, and $k_{\mathrm{temp}}=0.697$, according to the linear theory with one mode and two modes and numerical simulations (front-ligament pinching, FLP, and rear-ligament pinching, RLP). Deviations in the measured lengths are smaller than the size of the symbols. 


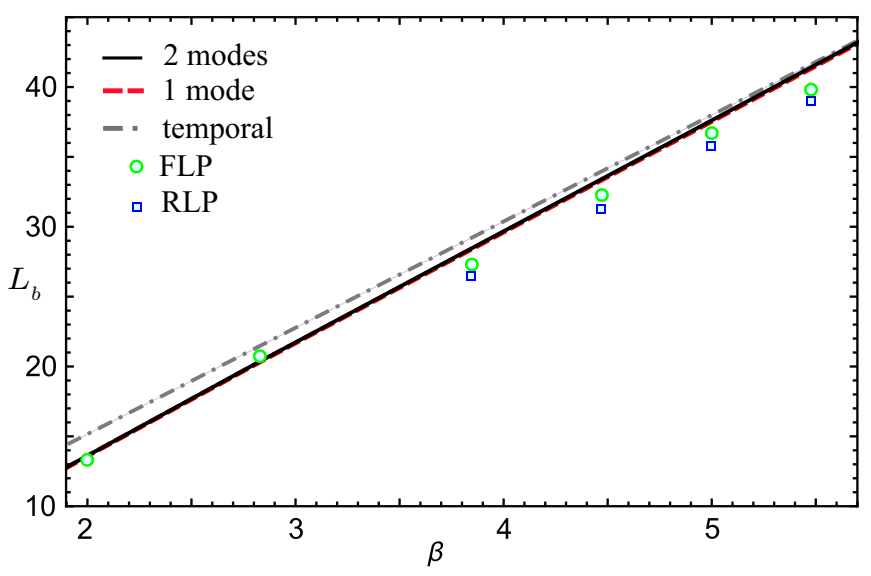

FIG. 5. Dimensionless breakup length vs the dimensionless jet velocity, $\beta=\sqrt{\text { We }}$. Symbols have the same meaning as in Fig. 4. Other parameters are fixed to $w_{0}=0.154, \mathrm{Oh}=0.01$, and $k_{\text {temp }}=0.697$.

where $L_{b}^{(1)}$ satisfies (11). After some manipulations and assuming $p \ll 1$ to retain only the relevant terms in Taylor expansions in $p$, we arrive at

$$
\Delta L_{b} \simeq \frac{1}{k_{\mathrm{d}, i}} e^{\left(k_{\mathrm{d}, i}-k_{\mathrm{s}, i}\right) L_{b}} .
$$

For the values of parameters selected to prepare Fig. 4, the estimate of $\Delta L_{b}$ provided by Eq. (12) predicts a deviation of some $10 \%$ for a breakup length $L_{b} \simeq 10$, which is in accordance with what this figure shows. However, a more revealing formula translates the relation between the breakup length and its deviation to a relation between the breakup time $t_{b} \equiv L_{b} / \beta$ and its deviation $\Delta t_{b} \equiv \Delta L_{b} / \beta$, recalling Eq. (9):

$$
\Delta t_{b} \simeq \frac{1}{\alpha_{\mathrm{d}}} e^{-\left(\alpha_{\mathrm{d}}-\alpha_{\mathrm{s}}\right) t_{b}}
$$

In this form, Eq. (13) shows clearly that as the breakup time is shorter, the deviation from the one-mode estimate is greater. To enter explicitly the factors that may cause this shortening, we can approximate $L_{b} \simeq L_{b}^{(1)}$ in the exponent in (12) to finally obtain

$$
\Delta L_{b} \simeq \frac{1}{2 \omega k_{\mathrm{d}, i}}\left|\frac{w_{0} k_{\mathrm{d}} k_{\mathrm{s}}}{k_{\mathrm{d}}-k_{\mathrm{s}}}\right|^{\left(k_{\mathrm{d}, i}-k_{\mathrm{s}, i}\right) / k_{\mathrm{d}, i}} .
$$

In the inviscid case, the exponent in (14) is 2 for any stimulation frequency [because $k_{\mathrm{s}, i}(\omega)=$ $-k_{\mathrm{d}, i}(\omega)$ ], but it increases with the Ohnesorge number and becomes dependent on the frequency.

The most important result of the spatial theory is the prediction of the breakup length for low Weber numbers, extending the findings of the temporal theory. This study is presented in Fig. 5, where we have fixed the perturbation amplitude to $w_{0}=0.15$, the dimensionless jet velocity $\beta$ is varied, and the same values of the remaining parameters are kept to those of Fig. 4. As in that previous figure, the two symbols correspond to front-ligament and rear-ligament breakup lengths numerically computed. For this moderate amplitude of perturbation, both the one-mode and the two-mode linear theories coincide and reproduce the numerical findings in the explored range of $\beta$.

A measure of the sensitivity of the jet to the velocity perturbations is the amplitude of the dominant mode per amplitude of perturbation, $f_{\mathrm{d}} / w_{0}$. In Fig. 6, this quantity is represented versus the stimulation frequency, measured through its corresponding wave number in the temporal approach, $k_{\text {temp }}=\omega / \beta$. This variable is a better choice than $\omega$ itself, as $0<k_{\text {temp }}<1$ always includes the unstable range independently of the Weber number. Several values of the Weber and Ohnesorge 


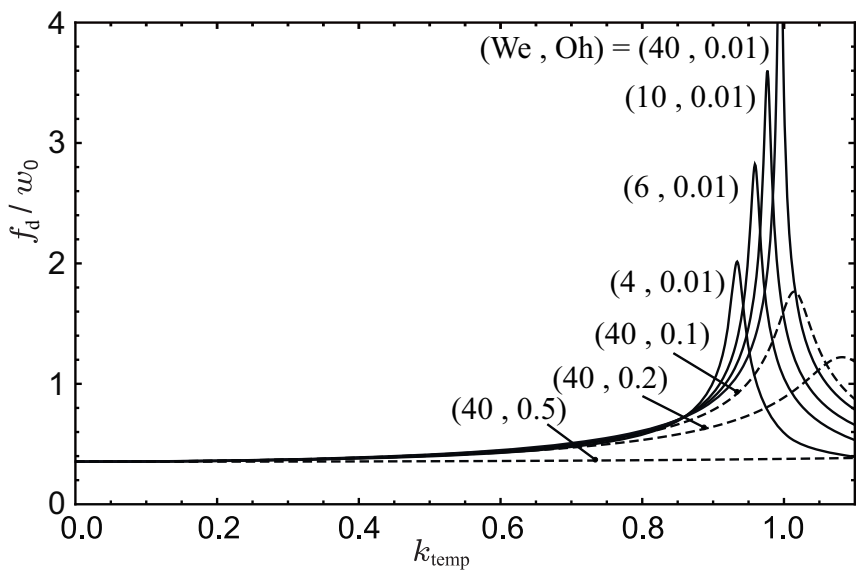

FIG. 6. Amplitude of the dominant capillary mode per amplitude of velocity perturbation, $f_{\mathrm{d}} / w_{0}$, as a function of the temporal wave number $k_{\mathrm{temp}}=\omega / \beta$, for different values of the Weber and Ohnesorge numbers.

numbers are selected to explore this sensitivity. Roughly speaking, wave numbers below $k_{\text {temp }} \simeq 0.7$ (a value typically near the maximum of the Rayleigh curves) give always similar values, in the approximate range $0.35<f_{\mathrm{d}} / w_{0}<0.5$, but beyond this point the sensitivity increases very rapidly, at least for low Ohnesorge numbers. The increase in the amplitude $f_{\mathrm{d}}$ is due to the diminishing of the term $k_{\mathrm{d}}-k_{\mathrm{s}}$ in the denominator of Eq. (7).

The above study in the perturbation frequency for the amplitude of the dominant mode per unit of perturbation amplitude has revealed a nonuniform response. If this response had been uniform, the minimum breakup length would be strictly associated with the maximum growth rate, but this is not the case. To explore where is the actual minimum, Fig. 7 shows computations of the breakup length versus the temporal wave number for three different amplitudes of stimulation, $w_{0}=0.05,0.1$, and 0.154. Numerical simulations for some representative frequencies are also provided. The agreement, to be discussed in the next section, is better for the lowest perturbation amplitude and for frequencies close to the maximum of the Rayleigh curve. As advanced, both theoretical curves and numerical

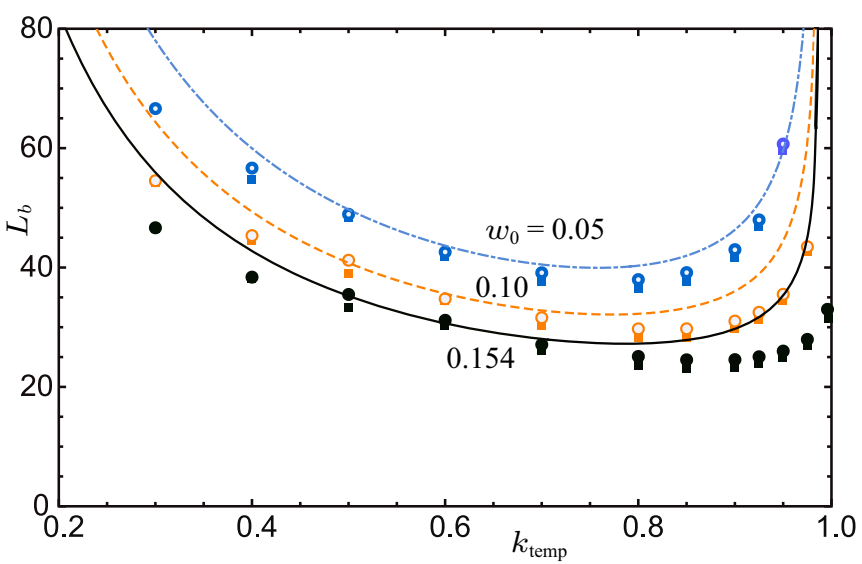

FIG. 7. Breakup length as a function of the temporal wave number $k_{\mathrm{temp}}=\omega / \beta$, for different values of the amplitude of velocity perturbation, $w_{0}$. The remaining parameters are $\mathrm{We}=14.8, \mathrm{Oh}=0.01$. Lines are the linear theory predictions and symbols are numerical results for front and end pinching as in previous figures. 


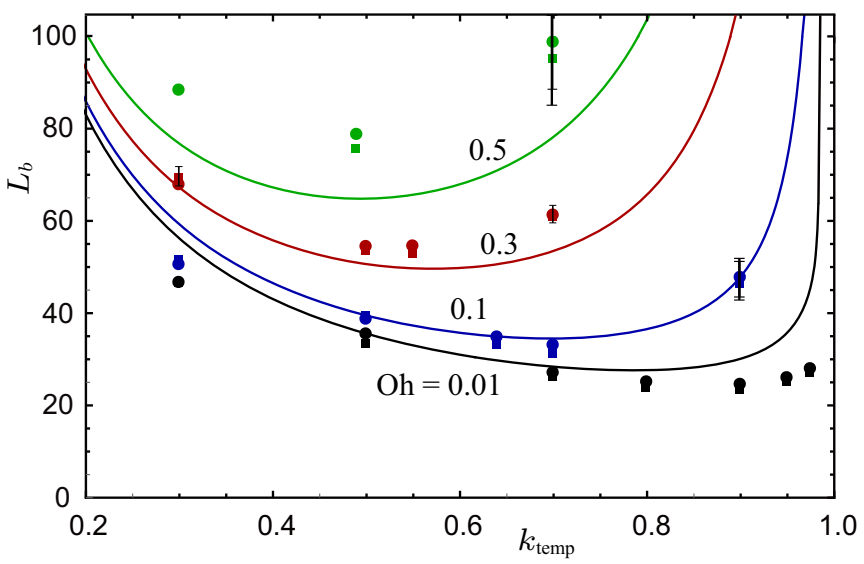

FIG. 8. Breakup length as a function of the temporal wave number $k_{\text {temp }}=\omega / \beta$, for different values of the Ohnesorge number. The remaining parameters are $\mathrm{We}=14.8, w_{0}=0.154$. Lines are the linear theory predictions and symbols are numerical results for front and end pinching as in previous figures. Only the vertical dispersion bars exceeding the dimensions of the points are plotted.

simulations show that the minimum in breakup length does not correspond to the frequency with maximum growth rate but to a greater frequency.

Finally, in Fig. 8 we have compared the predictions of the linear theory against numerical simulations with different Ohnesorge numbers. We can observe a progressive deviation between the numerical points and the theoretical curves as the Ohnesorge number increases.

\section{DISCUSSION}

The results shown in Fig. 3 demonstrate that the breakup length is quite insensitive to the radial dependence of the velocity perturbation profile, provided that the flow rate is fixed. As all the spatial modes discarded in our reduced model affect only this radial dependence but not the flow rate, we conclude that our model is justified. This explains why one-dimensional (1D) models, with simple radial dependence, give accurate predictions of the breakup length [16]. It has been demonstrated that these models describe well the two capillary modes, even for low Weber numbers [12].

The slope of a straight line fitted to the data of Fig. 4 allows measuring the growth rate of small-amplitude perturbations [21]. As expected, our linear theory predicts this growth rate well. This figure also illustrates that the two-mode model may be necessary to accurately predict the breakup length when the perturbations are strong enough. As mentioned in the previous section, the agreement is specially good for the pinching that occurs first in the ligament. This is a general rule that we may interpret as a consequence of the perturbing effect of the fast ligament recession, only affecting the second pinching event.

Equation (12) gives a simple estimate of the correction in breakup length due to the effect of the subdominant mode. Although the first reading of this formula can make us think that the correction $\Delta L_{b}$ depends essentially on the shortness of the intact length $L_{b}$, actually the breakup time through (13) is more relevant: As the breakup time shortens, the correction of the subdominant mode increases. This is particularly evident in Fig. 5, where values as low as $\beta=2$ are reached, giving intact lengths as low as $L_{b} / R \simeq 12$. However, for these lengths, the one-mode and two-mode theories agree, contrasting with the clear deviations shown in Fig. 4 for the same length. While the breakup length changes (proportionally to $\beta$ ), all the points in Fig. 5 have very similar nonsmall breakup times.

Another conclusion from the findings of the previous section is that there is not a strict correspondence between minimum intact length and maximum growth rate, as one usually tends to admit. Figure 7 shows what typically happens, both from the linear theory and from numerical 
simulations. Concerning the theoretical predictions, the shift of the minimum to a greater frequency is due to a significantly greater amplitude of the dominant mode for frequencies beyond the Rayleigh maximum, according to Fig. 6 . The theory also predicts the observed shift increase with $w_{0}$.

Concerning the deviation of the numerical results from the linear estimations in Fig. 7, these are small in the interval $0.5<k_{\text {temp }}<0.7$. If $k_{\text {temp }}$ is slightly greater or smaller than these limits, the discrepancy becomes greater, but independent of the initial amplitude $w_{0}$. As explained in Ref. [7], this is because the nonlinear evolution near breakup is independent of the initial amplitude of the perturbation whenever the initial transient of nonexponential growth finishes before the nonlinear terms are important. However, for sufficiently small or large $k_{\text {temp }}$, these deviations clearly depend on $w_{0}$ because the subdominant mode survives until the nonlinear terms begin to be significant, the initial transient becomes longer, and it overlaps the nonlinear growth regime. This is specially evident for $k_{\text {temp }}$ near 1 in Fig. 7.

The success of the linear predictions has restrictions regarding the Ohnesorge number. Indeed, Fig. 8 shows how increasing this parameter the difference between theory and numerical essays increases. However, these findings are not too surprising in view of the slow development of the long filaments between main drops observed in the simulations for $\mathrm{Oh}=0.3$ and 0.5 . The breakup in these cases are quite different from the simple growth of the dominant mode, implicit in the linear theory. The observed deviations are consistent with numerically computed breakup times reported by Ashgriz and Mashayek [22], which are greater than the temporal linear prediction for the maximum of the Rayleigh curve. The cited results refer to initial conditions of deformation-type instead of our impulse-type exit conditions, but both cases have the same trend. The failure of linear predictions should be taken into account if we are interested in a description of the natural (noisy) breakup length of very viscous jets, like in Ref. [23].

As pointed out in the introduction, the main goal of this work is to clarify how exit conditions must be handled in order to describe the jet evolution. Our reduced model, with only two modes, restricts the exit conditions to only two relevant parameters, namely, the amplitudes of the perturbation in the radius and in the mean axial velocity (complex in general). The connections between these parameters and the amplitudes of the two retained modes are natural and straightforward. On the other hand, a recent article about this subject proposes energy-based arguments for the same problem [8]. Let us analyze the shortcomings found in Ref. [8].

First, although the theory in Ref. [8] is dependent on the Weber number, their results are not constructed with a true spatial theory: (i) The authors use temporal growth rates obtained from the Rayleigh theory, and (ii) they apply the limit $\mathrm{We} \gg 1$ to deduce their exit conditions. Both assumptions are incompatible with considering Weber numbers as low as $\mathrm{We}=4$. Even their figure illustrating the role of the amplitude of velocity perturbation is constructed for a moderate value $\mathrm{We}=14.8$, for which the spatial theory predicts a significant deviation of the growth rate relative to their adopted temporal theory.

Second, they claim good agreement with numerically obtained data, shown in their Fig. 6, but a close inspection leads us to a very different scenario. Figure 9 gives a complete comparison of their findings and ours. Our numerical data (front-ligament and rear-ligament pinching estimates of the breakup length) and theoretical curves (spatial monomodal and spatial bimodal) have been extracted from Fig. 5, but adopting the way to measure the velocity perturbation in Ref. [8] by using $\delta$ (based on the jet velocity) instead of $w_{0}$ (based on the capillary velocity), i.e., $\delta=w_{0} / \beta$. It is evident that, besides the mentioned incongruence of the theoretical prediction of Moallemi et al. [8], we have been unable to reproduce their numerical results for the same boundary conditions and numerical parameters. Moreover, theoretical and numerical growth rates (inversely proportional to the slopes) do not agree. These facts contrast with the excellent agreement between our numerically computed data and our spatial theory, shown also in Fig. 4.

Also, Fig. 5 deserves comparison with Fig. 7 in Ref. [8]. Again, their numerical estimates of the breakup length for different Weber numbers are not reproducible and the agreement between theoretical and numerical findings is worse than ours. It should be mentioned that they fix $\delta=w_{0} / \beta$ (amplitude of the velocity perturbation relative to the jet velocity) instead of $w_{0}$ (same perturbation 


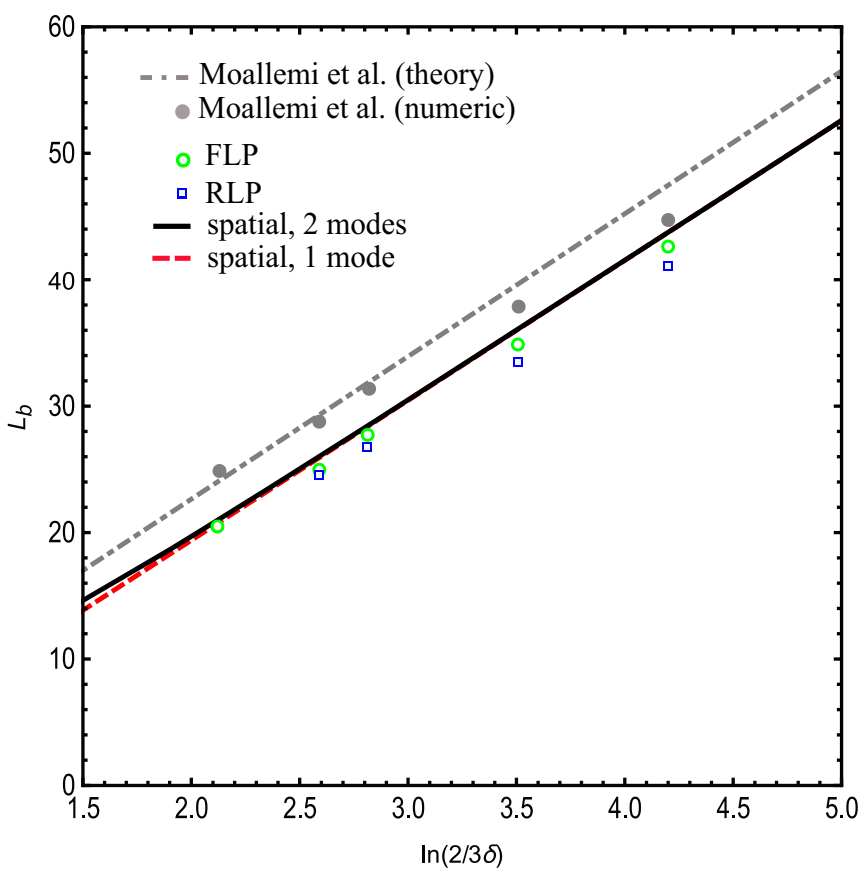

FIG. 9. Comparison between (i) numerical data and the linear theory extracted by Moallemi et al. [8], and (ii) numerical data and the two spatial linear theories (monomodal and bimodal) described in this work. As in Ref. [8], the dimensionless breakup length is represented vs $\ln (2 / 3 \delta)$, with $\delta$ being the mean axial velocity perturbation divided by the jet velocity, instead of $w_{0}$, which is made dimensionless with the capillary velocity.

relative to the capillary velocity). Therefore, for large enough $\beta$, their perturbations in velocity would become not so small and nonlinear effects would be significant. We emphasize that the best parameter to measure the perturbation in velocity is $w_{0}$, as the instability is driven by capillarity, not by inertia.

The above-mentioned incongruence of the results of Moallemi et al. [8] leads us to question how they estimate the equivalent radius modulation from the initial amplitude of the velocity perturbation. Their main assumption, namely that the rate of energy input through the orifice of both kinds of initial perturbations must be the same in order to yield the same evolution of the system, is not rigorously based on any conservation law, since no control volume is considered. Another mistake comes from assuming that their radius modulation evolves exponentially from the beginning. On the contrary, there is an initial transient of nonexponential growth of the surface deformation [7], not taken into account in their estimate of the breakup length. Finally, in their derivation of the general energy input, they use the condition $(\partial R / \partial x)_{x=0}=0$, where $R$ is the jet radius and $x$ is the axial coordinate (our $z$ ), which is valid only for the velocity disturbance case. All these facts explain the disagreement between theory and numerical simulations in Moallemi et al. [8]

\section{CONCLUSIONS}

Exit conditions for capillary jets with plug velocity profile must regard at least shape deformation and mean axial velocity perturbation. Numerical simulations have demonstrated that other features of the velocity perturbation are not essential to describe the jet spatial evolution. Among the infinitely many spatial modes, the two capillary modes are necessary and sufficient to formulate a reduced model for capillary jets subjected to the two mentioned natural exit conditions. As a consequence, the subdominant mode, although decaying, is necessary to construct the model. Even more, the subdominant mode gives, in some definite circumstances, significant corrections to the 
breakup-length predictions made with the dominant mode alone. The criterion to include this second mode deals with breakup times better than with breakup lengths: As the breakup time shortens, the subdominant-mode correction increases.

The performance of the proposed two-mode spatial linear model has been checked against numerical simulations based on the GERRIS code. The agreement is in some cases excellent, particularly when compared with the front pinching and for perturbation frequencies close to that corresponding to the maximum growth rate of the Rayleigh dispersion relation. The dependence of the amplitude of the dominant mode on the stimulation frequency explains why a stimulation with the frequency corresponding to the highest growth rate does not yield the shortest jet. Finally, as the Ohnesorge number increases, the linear predictions become worse, a fact not always taken into account in the recent literature about natural breakup length of very viscous jets.

\section{ACKNOWLEDGMENTS}

This work was supported by two Spanish public organizations: by the Ministerio de Economía, Industria y Competitividad, under Contract No. FIS2014-25161 and by the Junta de Andalucía under Contract No. P11-FQM-7919.

[1] O. A. Basaran, Small-scale free surface flows with breakup: Drop formation and emerging applications, AIChE J. 48, 1842 (2002).

[2] A. Barrero and I. G. Loscertales, Micro-and nanoparticles via capillary flows, Annu. Rev. Fluid Mech. 39, 89 (2007).

[3] J. Eggers, Nonlinear dynamics and breakup of free-surface flows, Rev. Mod. Phys. 69, 865 (1997).

[4] J. Eggers and E. Villermaux, Physics of liquid jets, Rep. Prog. Phys. 71, 1 (2008).

[5] L. Rayleigh, On the stability of jets, Proc. Lond. Math. Soc. 10, 4 (1878).

[6] L. Rayleigh, On the instability of a cylinder of viscous liquid under capillary forces, Philos. Mag. 34, 145 (1882).

[7] F. J. García and H. González, Normal-mode linear analysis and initial conditions of capillary jets, J. Fluid Mech. 602, 81 (2008).

[8] N. Moallemi, R. Li, and K. Mehravaran, Breakup of capillary jets with different disturbances, Phys. Fluids 28, 012101 (2016).

[9] L. Xie, L.-J. Yang, and H.-Y. Ye, Instability of gas-surrounded Rayleigh viscous jets: Weakly nonlinear analysis and numerical simulation, Phys. Fluids 29, 074101 (2017).

[10] J. B. Keller, S. I. Rubinow, and Y. O. Tu, Spatial instability of a jet, Phys. Fluids 16, 2052 (1973).

[11] S. J. Leib and M. E. Goldstein, Convective and absolute instability of a viscous jet, Phys. Fluids 29, 952 (1986).

[12] J. Guerrero, H. González, and F. J. García, Spatial modes of capillary jets, with application to surface stimulation, J. Fluid Mech. 702, 354 (2012).

[13] A. Sevilla, The effect of viscous relaxation on the spatiotemporal stability of capillary jets, J. Fluid Mech. 684, 204 (2011).

[14] F. J. García, H. González, J. R. Castrejón-Pita, and A. A. Castrejón-Pita, The breakup length of harmonically stimulated capillary jets, Appl. Phys. Lett. 105, 094104 (2014).

[15] A. Umemura, Self-destabilising loop of a low-speed water jet emanating from an orifice in microgravity, J. Fluid Mech. 797, 146 (2016).

[16] F. J. García and A. Castellanos, One-dimensional models for slender axisymmetric viscous liquid jets, Phys. Fluids 6, 2676 (1994).

[17] J. Gavis and M. Modan, Expansion and contraction of jets of Newtonian liquids in air: Effect of tube length, Phys. Fluids 10, 487 (1967). 
[18] S. Popinet, An accurate adaptive solver for surface-tension-driven interfacial flows, J. Comput. Phys. 228, 5838 (2009).

[19] A. U. Chen, P. K. Notz, and O. A. Basaran, Computational and Experimental Analysis of Pinch-Off and Scaling, Phys. Rev. Lett. 88, 174501 (2002).

[20] J. Eggers, Universal Pinching of 3D Axisymmetric Free-Surface Flow, Phys. Rev. Lett. 71, 3458 (1993).

[21] H. González and F. J. García, The measurement of growth rates in capillary jets, J. Fluid Mech. 619, 179 (2009).

[22] N. Ashgriz and F. Mashayek, Temporal analysis of capillary jet breakup, J. Fluid Mech. 291, 163 (1995).

[23] A. Javadi, J. Eggers, D. Bonn, M. Habibi, and N. M. Ribe, Delayed Capillary Breakup of Falling Viscous Jets, Phys. Rev. Lett. 110, 179902 (2013). 